\title{
Development of High Energy Particle Detector for the Study of Space Radiation Storm
}

\author{
Gyeong-Bok Jo', Jongdae Sohn², Cheong Rim Choi ${ }^{2}$, Yu Yi ${ }^{1 \dagger}$, Kyoung-Wook Min², \\ Suk-Bin Kang ${ }^{2}$, Go Woon $\mathrm{Na}^{3}$, Goo-Hwan Shin ${ }^{4}$ \\ ${ }^{1}$ Department of Astronomy and Space Science, Chungnam National University, Daejeon 305-764, Korea \\ ${ }^{2}$ Department of Physics, Korea Advanced Institute of Science and Technology, Daejeon 305-701, Korea \\ ${ }^{3}$ Department of Physics, Ehwa Womans University, Seoul 120-750, Korea \\ ${ }^{4}$ Satellite Technology Research Center, Korea Advanced Institute of Science and Technology, Daejon 305-701, Korea
}

Next Generation Small Satellite-1 (NEXTSat-1) is scheduled to launch in 2017 and Instruments for the Study of Space Storm (ISSS) is planned to be onboard the NEXTSat-1. High Energy Particle Detector (HEPD) is one of the equipment comprising ISSS and the main objective of HEPD is to measure the high energy particles streaming into the Earth radiation belt during the event of a space storm, especially, electrons and protons, to obtain the flux information of those particles. For the design of HEPD, the Geometrical Factor was calculated to be 0.05 to be consistent with the targets of measurement and the structure of telescope with field of view of $33.4^{\circ}$ was designed using this factor. In order to decide the thickness of the detector sensor and the classification of the detection channels, a simulation was performed using GEANT4. Based on the simulation results, two silicon detectors with $1 \mathrm{~mm}$ thickness were selected and the aluminum foil of $0.05 \mathrm{~mm}$ is placed right in front of the silicon detectors to shield low energy particles. The detection channels are divided into an electron channel and two proton channels based on the measured LET of the particle. If the measured LET is less than $0.8 \mathrm{MeV}$, the particle belongs to the electron channel, otherwise it belongs to proton channels. HEPD is installed in the direction of $0^{\circ}, 45^{\circ}, 90^{\circ}$ against the along-track of a satellite to enable the efficient measurement of high energy particles. HEPD detects electrons with the energy of $0.1 \mathrm{MeV}$ to several $\mathrm{MeV}$ and protons with the energy of more than a few MeV. Thus, the study on the dynamic mechanism of these particles in the Earth radiation belt will be performed.

Keywords: instruments for the study of space storm, space radiation particle, high energy particle detector

\section{INTRODUCTION}

Next Generation Small Satellite-1 (NEXTSat-1) is scheduled to launch in 2017 and Instruments for the Study of Space Storm (ISSS) is planned to be onboard the NEXTSat-1. High Energy Particle Detector (HEPD) is one of the equipment comprising ISSS and the HEPD measures the high energy electrons and protons streaming into the Earth radiation belt during the event of a space storm in a space radiation environment. The study on the capture and annihilation of the high energy particles will be performed based on the measurement of particles through this equipment. Space storms indicate perturbations of nearearth space environment due to rapid changes in solar activity, such as Coronal Mass Ejection (CME) or Solar Flare. The space storms accompany various phenomena such as the increase of radiation particles in the Earth radiation belt and northern lights in the polar region. Among these space storms, the space storm resulting from the high speed solar wind when the Co-rotating Interaction Region (CIR) passes the magnetosphere usually occurs at the declining phase of solar activity. It is not so fatal compared to the (c) This is an Open Access article distributed under the terms of the Creative Commons Attribution Non-Commercial License (http:// creativecommons.org/licenses/by-nc/3.0/) which permits unrestricted non-commercial use, distribution, and reproduction in any medium, provided the original work is properly cited.
Received Aug 29, 2014 Revised Sep 4, 2014 Accepted Sep 5, 2014 †Corresponding Author

E-mail: euyiyu@cnu.ac.kr Tel: +82-42-821-5468, +82-42-821-8891 
space storm during solar maximum, but has more impact on the Earth radiation belt due to the higher frequency of event (Choi et al. 2014). The NEXTSat-1 will be launched in 2017 when it is solar declining phase rather than a solar maximum. Thus, the measurement of space storms will lead to significant accomplishments in the researches. Also, since it will rotate along the Sun-synchronous polar orbit, the collaborating researches with measurement mission satellites on the orbit of equatorial plane, such as Van Allen Probe and Energization and Radiation in Geospace (ERG), are expected to produce more fruitful results (Sohn et al. 2012). The Earth radiation belt was first discovered in 1958 by Explorer 1 and it has been a continuous subject of researches in view of fundamental science and engineering since the discovery. From the perspective of fundamental science, there have been researches on the mechanism of the origination, capture, and annihilation of high energy particles, while in view of engineering, there have been studies on the impact of these particles on the human space activities (Van Allen et al. 1958). Especially, in this age of space, the concerns on the impacts of high energy particles on a spacecraft and astronauts have begun to emerge. Among those, the killer electrons with energy of about $100 \mathrm{keV}$ are considered to be the main cause of failures in satellites and spacecrafts, which are considered to be a great obstacle to overcome for the human exploration of space. Thus, the measurement of high energy particles in the Earth radiation belt during the event of space storm will be a research topic of fundamental science like the mechanism of particle acceleration and also will be an engineering basis for the human space exploration.

In this paper, the design of HEPD which is one of the equipment of ISSS onboard the NEXTSat-1 launching in 2017, is described. The measurement targets of HEPD are electrons with energy of $0.1 \mathrm{MeV}$ to a few $\mathrm{MeV}$ and protons with energy of more than several $\mathrm{MeV}$ also with the maximum particle flux of $\sim 10^{6} /\left(\mathrm{cm}^{2} \cdot \mathrm{s}\right)$.

\section{MECHANICAL STRUCTURE}

\subsection{HEPD Telescope Design}

It is necessary to calculate the appropriate Geometrical Factor which is consistent with the measurement targets in order to determine the mechanical structure of the HEPD telescope. The Geometrical Factor is the proportionality coefficient between the counting rate and the incident particle flux when the incident particles show isotropic behavior. The magnitude of the Geometrical Factor is determined by the geometrical structure of a telescope. In other words, if we could calculate the Geometrical Factor using the correlation between the counting rate and the flux, we can decide the mechanical structure of a telescope. The correlation between counting rate(C) and Flux(I) can be described as follows.

$$
\mathrm{C}=\mathrm{G} \times \mathrm{I} \text {, }
$$

where $\mathrm{C}, \mathrm{G}$, and I are the counting rate, the Geometrical Factor, and the flux of incident particles, respectively. The counting rate in this study is determined to be $10^{5} / \mathrm{s}$ which is the design target of the HEPD. According to the AE-8/AP-8 model (Schiller et al.,2010) with $\mathrm{L}=4$, the fluxes are obtained as below.

For electrons

$$
\mathrm{I}(\mathrm{E})=3.003 \times 10^{5} \times E^{-2.3028}
$$

For protons

$\mathrm{I}(\mathrm{E})= \begin{cases}5.2008 \times 10^{4} \times E^{-1.1682} & (0 . \leq E \leq 26 \mathrm{MeV}) \\ 9.6489 \times 10^{8} \times E^{-4.2261} & (26 \mathrm{MeV} \leq E)\end{cases}$

For the calculation of Geometrical Factor, the maximum flux in the measurement energy range of HEPD, $2.0 \times 10^{6}(/$ $\mathrm{cm}^{2} \cdot \mathrm{sr} \cdot \mathrm{s}$ ), is used to obtain the following result.

$$
\mathrm{G}=\frac{C}{I}=\frac{10^{5}}{2.0 \times 10^{6}}=0.05
$$

where the unit of Geometrical Factor is $\mathrm{cm}^{2} \cdot \mathrm{sr}$. The Geometrical Factor in a cylindrical geometry (Fig. 1) can be expressed by the following equation (Sullivan 1971):

$\mathrm{G}=\frac{1}{2} \pi^{2}\left[R_{1}{ }^{2}+R_{2}{ }^{2}+h^{2}-\sqrt{\left[\left(R_{1}-R_{2}\right)^{2}+h^{2}\right]\left[\left(R_{1}+R_{2}\right)^{2}+h^{2}\right]}\right]$.

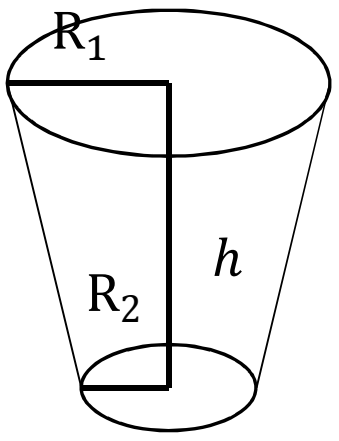

Fig. 1. Parameters of a cylindrical telescope. 


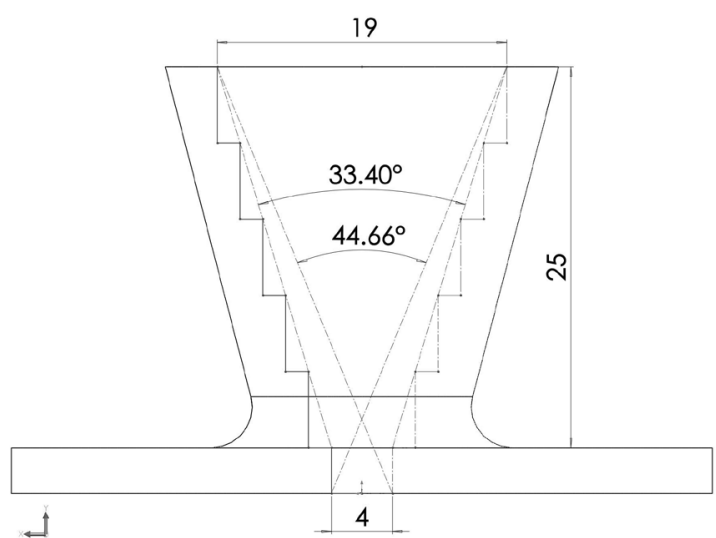

Fig. 2. HEPD telescope design.

Using Eq. (5), the values of $R_{1}, R_{2}, h$ corresponding to $\mathrm{G}=0.05$ are calculated to be $R_{1}=9.5(\mathrm{~mm}), R_{2}=2(\mathrm{~mm}), h$ $=25(\mathrm{~mm})$ and the telescope design of HEPD is shown in Fig. 2. The telescope of this design has the Field of View of $33.4^{\circ}$ and it is installed in the direction of $0^{\circ}, 45^{\circ}, 90^{\circ}$ against the along-track of satellite like Fig. 3 to enable the efficient measurement of high energy particles.

\subsection{HEPD Sensor Design}

HEPD analyzes particles by measuring Linear Energy Transfer (LET) which is generated when they pass the sensor. When a charged particle is incident on a sensor, some of the particle energy is lost due to the interactions with the medium. The amount of energy loss per unit path length is called LET. The LET generated by the particles in the HEPD energy spectrum is governed by the following Bethe formula (Bethe \& Ashkin 1954).

$$
-\frac{d E}{d x}=4 \pi r_{0}^{2} \frac{m c^{2}}{\beta^{2}} N Z\left[\left(\ln \frac{2 m c^{2}}{\bar{I}} \beta^{2}\right)-\ln \left(1-\beta^{2}\right)-\beta^{2}\right]
$$

where $r_{0}$ : classcal electro radius, $\beta=\frac{v}{c}, \mathrm{~N}$ : atom density of material, and $\mathrm{Z}$ : atom number of material. The energy loss of a particle which travels in a medium along a path length of $t$ can be calculated by the following equation using the LET.

$$
\Delta \mathrm{E}=\left(-\frac{d E}{d x}\right)_{0} t
$$

The energy loss of the particle is converted into an electron-hole pair to generate electric signal in the detector sensor. Since this signal is proportional to the amount of energy transferred to the sensor, the energy loss of the particle can be obtained by analyzing this signal.

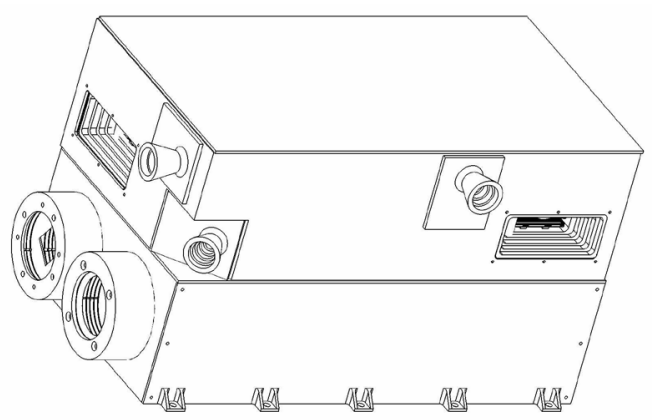

$\leftrightarrow$

Fig.3. ISSS Mechanical structure.

\subsubsection{GEANT4 Simulation Result}

The LET shows different behaviors according to the species of incident particles, energy of the particle, medium property, and sensor thickness. The mission of HEPD silicon detector is to detect high energy electrons and protons. Thus, a GEANT4 simulation was performed in order to find out the LET of a particle depending on the variation of the silicon thickness. In Fig. 4, it is shown that the LET increases as the silicon thickness increases. That is, the thicker the sensor is, the higher the detected energy of a particle is. However, for more efficient detection of particles, it was decided to use two sensors with silicon thickness of $1 \mathrm{~mm}$. Also, an aluminum foil of $0.05 \mathrm{~mm}$ thickness is placed right in front of the silicon sensor to shield the low energy particles with energy less than the target. In Fig.5, the HEPD Sensor is adumbrated and Fig. 6 shows the entire structure and the internal cross section of HEPD with the sensors installed. In order to determine the detection channels, LET simulations were performed for the entire structure of HEPD. In Fig. 7, it was shown that electrons of energy less than $0.1 \mathrm{MeV}$ and protons of energy less than 2 $\mathrm{MeV}$ were shielded by $0.05 \mathrm{~mm}$ aluminum foil and each silicon sensor could measure electrons of energy up to $0.8 \mathrm{MeV}$ and protons of energy up to $12 \mathrm{MeV}$. According to this result, those particles of the LET measured less than $0.8 \mathrm{MeV}$ are classified as the electron channel and those particles of LET greater than $0.8 \mathrm{MeV}$ are classified as two proton channels to make up total of 3 channels. The detection channel for electrons will be subdivided using the Look Up Table.

\subsubsection{Proton-electron Contamination}

Looking into detection spectrum more closely, it is revealed that electrons are detected below $0.8 \mathrm{MeV}$ and 


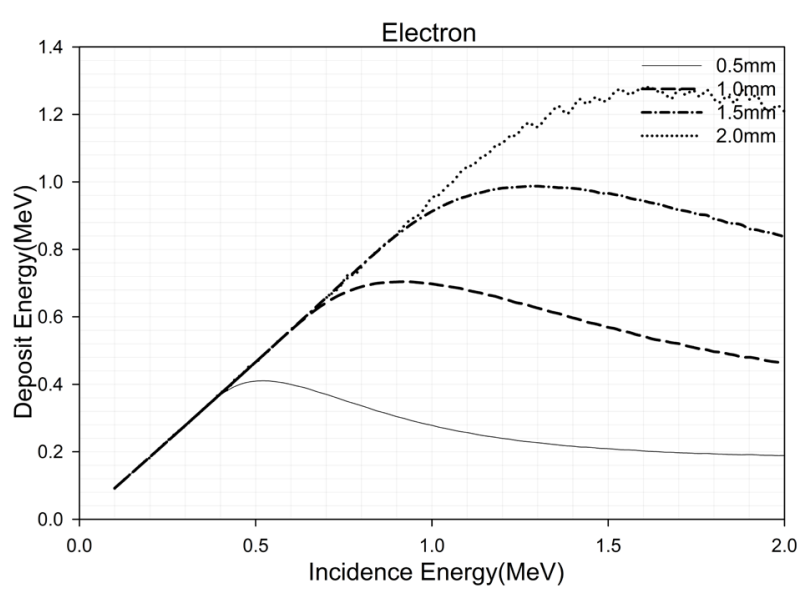

(a)

Fig. 4. LET change according to silicon thickness (a) electron (b) proton.

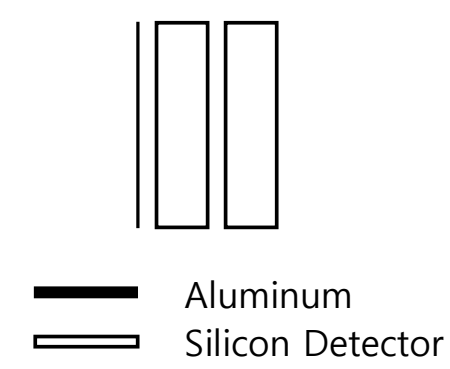

Fig. 5. Aluminum and silicon detector placement.

protons are detected in the range of 0 to $12 \mathrm{MeV}$ on a basis of the measured LET in each SSD. Thus, if the LET of a particle is greater than $0.8 \mathrm{MeV}$, it must be a proton, however, for the particles of LET between 0 and $0.8 \mathrm{MeV}$, it

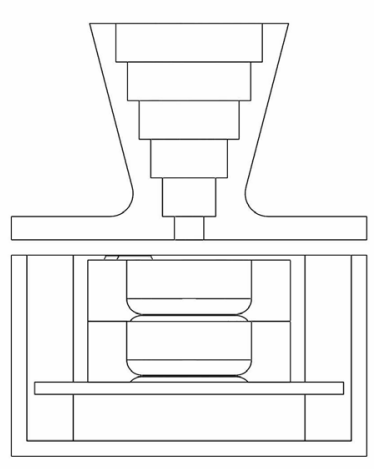

$\stackrel{2}{\longleftrightarrow}$

(a)

Fig. 6. (a) HEPD internal cross section, (b) HEPD entire structure.

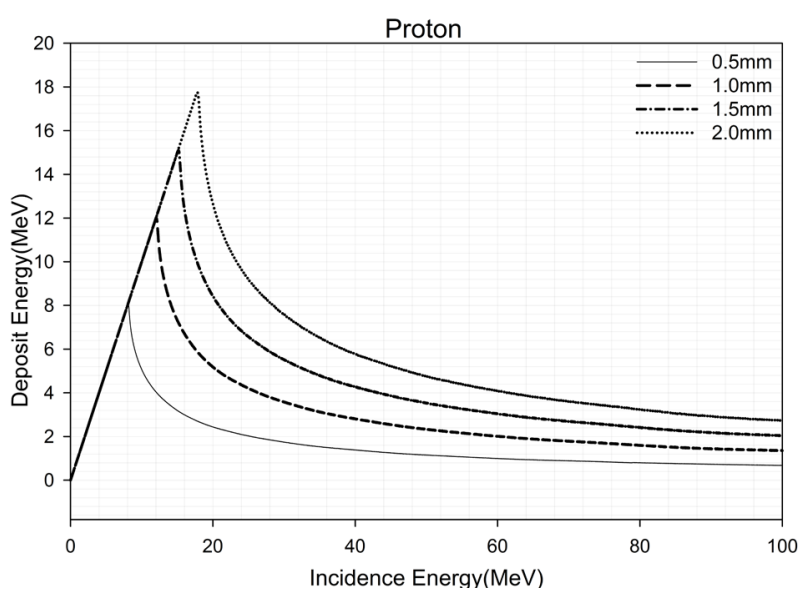

(b)

is difficult to determine whether the particle is a proton or an electron due to proton-electron contamination issue. In order to address this concern, the forementioned flux and LETs resulting from the particle passing the silicon sensor of $1 \mathrm{~mm}$ thickness were compared. The LET results are shown in Fig. 8. The fluxes of electron and proton in the sky over the equator are obtained using Eq. (2) and (3) based on the AE/AP-8 model with $\mathrm{L}=4$. Combining the above two results to plot particle flux versus LET gives the following results. As shown in Fig. 10, the maximum LET of electrons is about $0.8 \mathrm{MeV}$. That is, when the measured LET is greater than 0.8 $\mathrm{MeV}$, only protons are detected and the flux in this range is considered to be a proton flux. For the LET less than 0.8 $\mathrm{MeV}$, the proton flux can be estimated by extrapolating the proton flux of LET greater than $0.8 \mathrm{MeV}$. The proton flux obtained is subtracted from the total flux measured in the

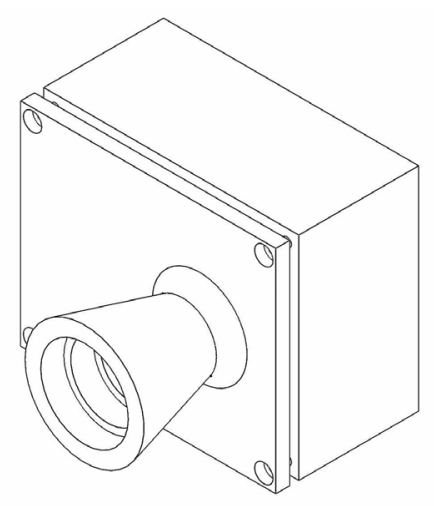

2. (b) 


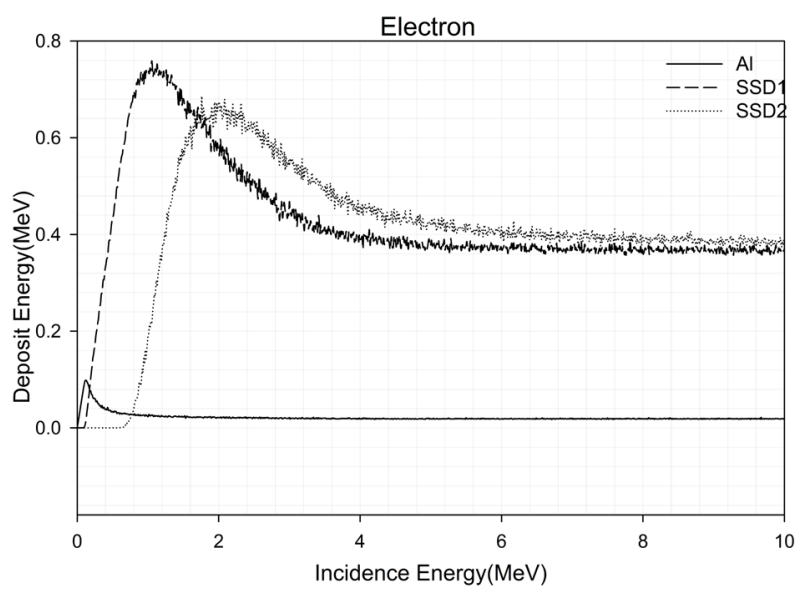

(a)

Fig. 7. LET change of whole HEPD: (a) electron and (b) proton.

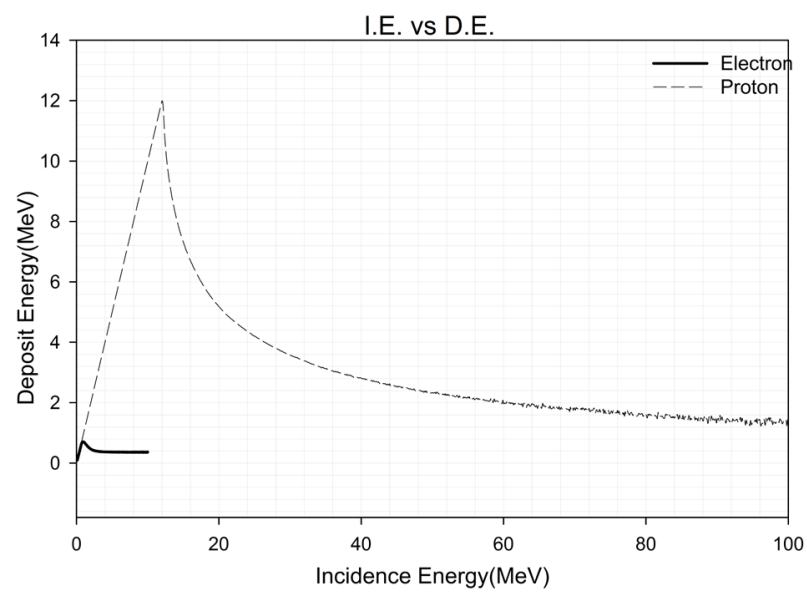

Fig. 8. LET change of protons and electron in a $1 \mathrm{~mm} \mathrm{SSD}$.

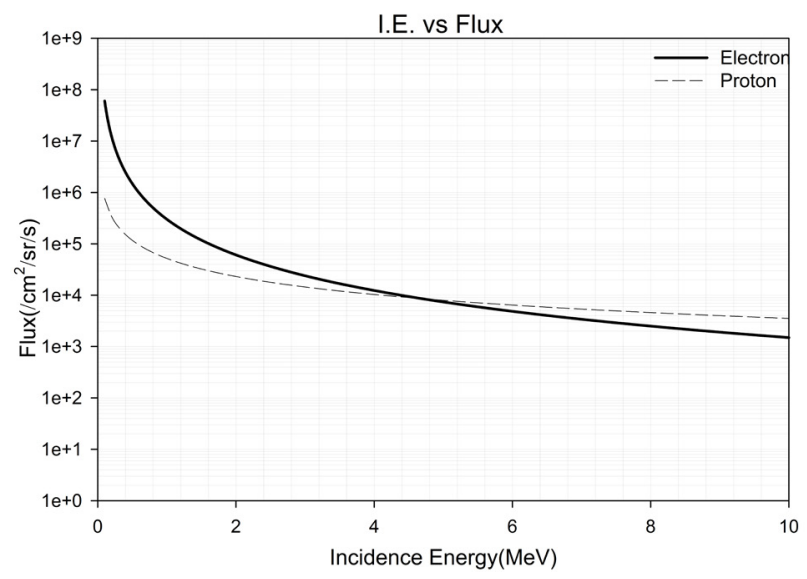

Fig. 9. $0 \sim 10 \mathrm{MeV}$ electron \& proton Flux.

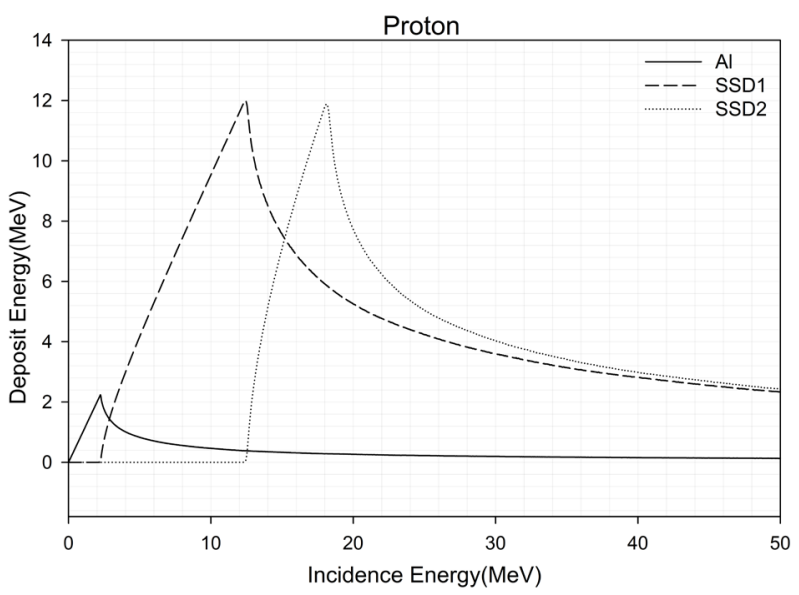

(b)

range less than $0.8 \mathrm{MeV}$ to produce the electron flux. Using this approach, the proton-electron contamination can be resolved in each SSD of HEPD.

\section{SYSTEM BLOCK DIAGRAM}

The entire HEPD system is divided into 3 parts of Preamp board, Shaping board, and Control board. The Preamp board converts the energy of an incident particle into an electric signal and amplify it. The Shaping board converts this signal into data. Then the data from the Shaping board is digitized on the Control board and this Control board supplies the power to the entire HEPD and checks the functions of HEPD. Also the Control board manages and controls the overall operation of HEPD.

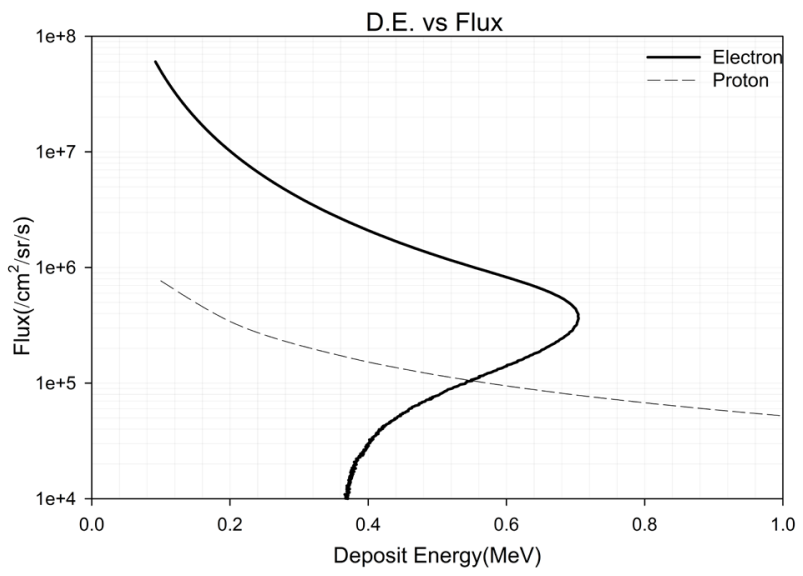

Fig. 10. Relation of Flux and LET on SSD $1 \mathrm{~mm}$. 


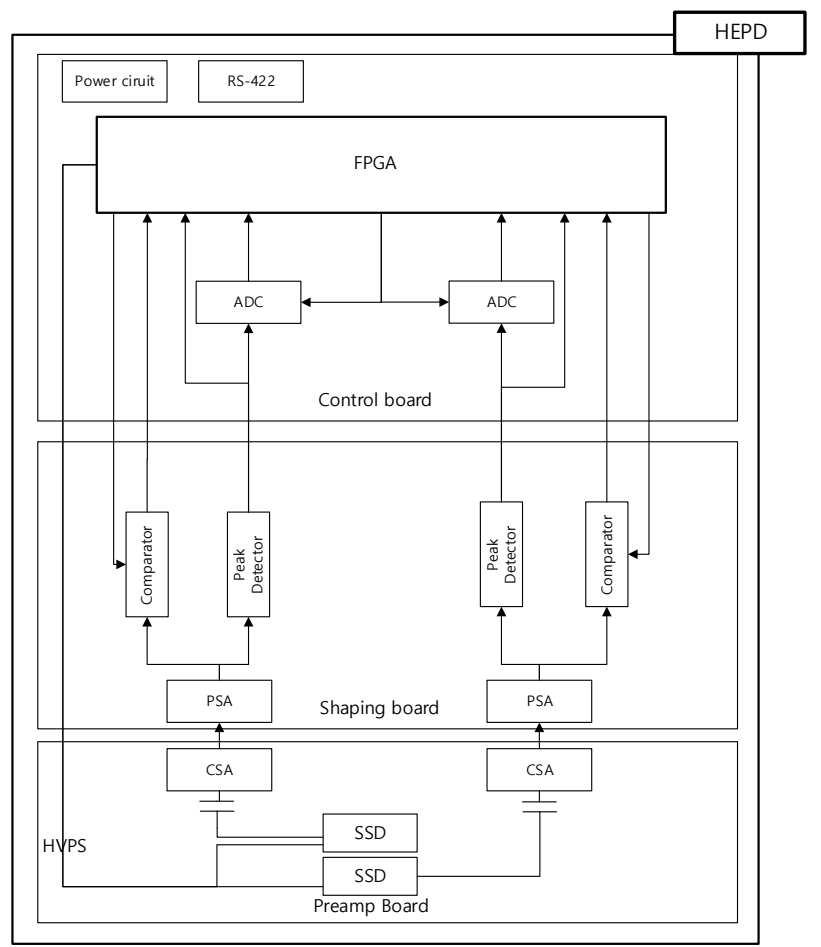

Fig. 11. HEPD system block diagram.

\subsection{Preamp Board}

Preamp board consists of a silicon detector and a Charge Signal Amplifier (CSA). The silicon detector converts the energy lost when a particle passes the sensor into an electric signal. The thicker the silicon detector is, the larger the amount of energy loss of the particle. Fully depleted silicon surface barrier detector of Ortec corporation is used for the silicon detector. The CSA amplifies a signal from the silicon detector and A250F-NF of Amptek corporation is selected for the CSA.

\subsection{Shaping Board}

In the Shaping board, a Pulse Shaping Amplifier (PSA) converts the electric charge signal from the Preamp board into a pulse signal in Gaussian shape and analyzes this signal to generate data. A275FC of Amptek corporation is used for the PSA and Baseline Restorer is used to maintain the GND of the amplifier constant. Also, a comparator and a peak detector are used to generate the data from the pulse signal.

\subsection{Control Board}

The Control board digitize the data from the Shaping board and manages the entire HEPD. The pulse height signals from the Shaping board are converted into digital signals through ADC. Also the control board supplies power to all the components of HEPD and performs the overall management of HEPD system operation in the FPGA.

\section{DISCUSSION AND SUMMARY}

HEPD is one of the equipments comprising ISSS onboard the NEXTSat- 1 launching in 2017 and is under development. The HEPD measures the high energy electrons and protons streaming into the Earth radiation belt during the event of a space radiation storm in order to obtain the information on the flux depending on the species and the energy of a particle. In order to design HEPD, the maximum flux of the observation target in the sky over the equator was obtained to be $2.0 \times 10^{6}\left(/ \mathrm{cm}^{2} \cdot \mathrm{sr} \cdot \mathrm{s}\right)$ using the AE-8/AP-8 model with $\mathrm{L}=4$. Also using the counting rate of $10^{5} / \mathrm{s}$ which is the design target of the HEPD, the HEPD Geometrical Factor is determined to be $0.05\left(\mathrm{~cm}^{2} \cdot \mathrm{sr}\right)$. Based on this value, the structure of HEPD telescope was determined to have the Field of View of $33.4^{\circ}$. Also, the GEANT4 simulation was performed to determine the structure of HEPD sensor. According to the simulation results, it was decided to use two sensors with silicon thickness of $1 \mathrm{~mm}$. Also, an aluminum foil of $0.05 \mathrm{~mm}$ thickness is placed right in front of the silicon sensor to prevent the incidence of low energy particles. In order to distinguish electrons from protons, the approach to remove proton flux from the total measured flux considering the flux difference of each particle depending on LET was used. Also, LET simulations for entire HEPD sensors were performed. Based on the results of the simulation, those particles with measured LET less than $0.8 \mathrm{MeV}$ belong to the electron channel and those over $0.8 \mathrm{MeV}$ belong to two proton channels to comprise total of 3 channels. The detection channel for electrons will be subdivided using the Look Up Table.

Using the information obtained in this way, the study on the mechanism of capture and annihilation of the particles in the Earth radiation belt will be performed in the future. Especially, the NEXTSat-1 will have a measurement mission rotating along the Sun-synchronous polar orbit. Thus, the collaborating researches with other foreign satellites on the orbit of equatorial plane, such as Van Allen Probe, ERG, or THEMIS, are expected to produce many fruitful results in the study of the radiation belt. 


\section{ACKNOWLEDGEMENTS}

We acknowledge the support by the National Research Foundation of Korea through its grant (2012M1A3A4A01056418).

\section{REFERENCES}

Bethe AH, Ashkin J, Passage of Radiations through Matter in Experimental Nuclear Physics, Vol. 3, ed. Segrè E (Wiley, New York, 1953), 166-357.

Choi CR, Sohn JD, Lee JC, Seo YM, Kang SB, et al., Scientific Missions and Technologies of the ISSS on board the NEXTSat-1, JASS, 31, 73-81(2014). http://dx.doi. org/10.5140/JASS.2014.31.1.73

Schiller QG, Mahendarkumar A, Li X., REPTILE: A Miniaturized Detector for a CubeSat Mission to Measure Relativistic Particles in Near-Earth Space, 24th Small Satellite Conference, AIAA/USU, Logan, UT, 9-10 Aug (2010)

Sohn JD, Oh SY, , Yi Y, Min KW, Lee DY, et al., A Design of Solar Proton Telescope for Next Generatioin Small Satellite, JASS, 29, 343-349(2012). http://dx.doi. org/10.5140/JASS.2012.29.4.343

Sullivan JD, Geometrical factor and directional response of single and multi-element particle telescope, NucIM, 95, 5-11 (1971). http://dx.doi.org/10.1016/0029$554 X(71) 90033-4$

Van Allen JA, Ludwig GH, Ray EC, Mcllwain CE, Observation of high intensity radiation by satellites 1958 Alpha and Gamma, Jet Propul, 28, 588-592 (1958). 